results section, we split up descriptive information from primary studies (sex rates) and analytic approaches (considering sex/gender in the assessment of risk of bias, presenting disaggregated data by sex/gender or subgroup or heterogeneity analyses). We used 'not applicable' to denote a situation where insufficient primary studies or data on estimates did not enable to conduct the intended analyses (e.g. meta-analysis, subgroup analysis). We performed descriptive statistics and regression analyses to assess associations between authors' gender and sex/gender reporting.

Results 556 reviews were screened, of which 91 were excluded due to withdrawal $(19,8 \%)$ or sex-specific disease (80,2\%). Our analysis comprised 465 studies, including 2 prognosis, 4 methodology, 5 overview, 20 diagnostic and 421 intervention reviews. Women represented 53,8\% ( $\mathrm{n}=250)$ and $38,9 \%(\mathrm{n}=181)$ of first and last authorship, respectively, while in $25,3 \%$ of reviews both authors were women. $85,6 \%$ of authors belonged to high-income countries. 7.5\% ( $n=35)$ of reviews reported on sex in the abstract, 17.6\% ( $n=82)$ in the methods and 61.4\% $(\mathrm{n}=285)$ in the results section. Of these 285, 64.7\% provided descriptive results and 16,3\% had an analytic approach. In the discussion section, $13.5 \%$ (n $=63$ ) of reviews addresses sex-related findings. Only 4 studies scored positive in all 4 sections. Studies with female firstlast authorship had a non-significant increased probability of reporting on sex (RR [95\% CI] 1,24 [0.68-1.92])

Conclusions Consideration of sex and gender in Cochrane reviews is scarce. This prevents from generating inclusive and unbiased health research and inhibits effective population-wide translation of results. While women are less likely to be last authors, studies with a female first or last author show an increased (although non-significant) probability of reporting on sex.

\section{PSYCHOSOCIAL CONSEQUENCES OF INVITATION TO COLORECTAL CANCER SCREENING - A DANISH LONGITUDINAL COHORT STUDY}

\footnotetext{
1,2 2 essica Malmqvist, 'Volkert Siersma, 'Mie Sara Hestbech, 'Dagný Rós Nicolaisdóttir, ${ }^{3}$ Christine Winther Bang, ${ }^{2,3}$ John Brodersen. ${ }^{1}$ Center for Research and Education in General Practice, Copenhagen, Denmark; 'Research Unit for General Practice in Region Zealand, Copenhagen, Denmark; ${ }^{3}$ The Center for Research and Education in General Practice, Copenhagen, Denmark
}

\subsection{6/bmjebm-2019-EBMLive.36}

Objectives Harms of screening are generally not well reported. Harms of colorectal cancer (CRC) screening may include negative psychosocial consequences from the attention being drawn to disease through the screening invitation, the screening procedure itself and from fear of the screening result. Hence, it is important to measure psychosocial consequences before and after invitation to screening, at screening and after screening. Moreover, measurement of psychosocial consequences in cancer screening settings should be performed using questionnaires with high content validity and adequate measurement properties. Despite this fact, few CRC screening studies investigating psychosocial consequences have performed a baseline measurement before and after invitation to screening in both a screening and a control cohort. Moreover, none of the studies has used a questionnaire with high content validity and adequate measurement properties.

Therefore, the objective of this study was to investigate the psychosocial consequences of invitation to CRC screening in a
Danish CRC screening naïve cohort using a condition-specific questionnaire validated with Item Response Theory Rasch Models.

Method A random sample of 1000 screening invitees scheduled for screening in March 2017 was matched with 1000 control persons scheduled for screening in November and December 2017. We sent a questionnaire to both study groups five weeks prior to the invitation of the invitees and another questionnaire to both groups three days after the invitees' screening invitations were sent by post.

The primary outcome was psychosocial status, measured with the condition-specific questionnaire Consequences of Screening for CRC (COS-CRC).

We analysed the mean COS-CRC score at each measurement for each COS-CRC scale compared between the study groups using multivariable regression models, adjusting for potential confounders. We adjusted for differential nonresponse by weighting the observations that were available at the follow-up measurement by the inverse of the probability of not being missing. We also adjusted for repeated measures and weighting. To allow for multiple testing, a p-value $<0.01$ was considered significant.

Results Results and conclusions will be presented at the conference.

Conclusions Results and conclusions will be presented at the conference.

\section{INCIDENCE OF SUBSTUDIES PUBLICATION AFTER ORIGINALLY NEGATIVE CLINICAL TRIALS AND RATE OF POSITIVATION: A CALL FOR SCIENTIFIC INTEGRITY}

1,2Luis Correia, 'Diego Rabelo, 'Alessandra Caldas, 'Bruno Góes, 'Janine Magalhães, ${ }^{3}$ Daniel Medina, 'Denise Matias. 'Bahiana Medical and Public Health School, Salvador, Brazil; ${ }^{2}$ Hospital São Rafael, D'Or Institute for Research and Education, Salvador, Brazil; ${ }^{3}$ School of Medicine, Federal University of Bahia, Santo Antonio de Jesus, Brazil

\subsection{6/bmjebm-2019-EBMLive.37}

Objectives After a negative study, substudies may emerge providing a positive spin on the hypothesis, based on subgroup or secondary outcome analyses. This approach suffers from multiplicity problem, imprecision and higher risk of type I error.

To describe the incidence of substudies testing the same hypothesis over a period of 5 years after publication of a primarily negative randomized clinical trial; to access the most frequent subanalysis method, how explicit the exploratory nature of the analysis were and the rate of successful positivation of results.

Method We searched all negative randomized trials published in the New England Journal of Medicine (NEJM) during the year 2014. Then, we made a highly sensible PUBMED search over the following 5 years in order to detect subsequent articles testing the same hypothesis in the same dataset throughout secondary analysis. Methods utilized, lack of recognition as secondary analysis was described and rate of positivation were described.

Results During the year 2014, 46 negative randomized clinical trials were published in the NEJM. Over five years, 14 of those articles had subsequent publication of subanalysis in PUBMED indexed journals, an incidence of 30\% $(95 \% \mathrm{CI}=$ $19 \%$ - 45\%). The most frequent method was secondary endpoints analysis (67\% of substudies) and the remaining made use of subgroup analysis. Half of those analysis were not 